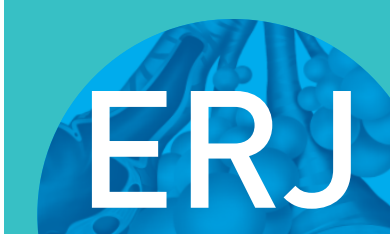

open research
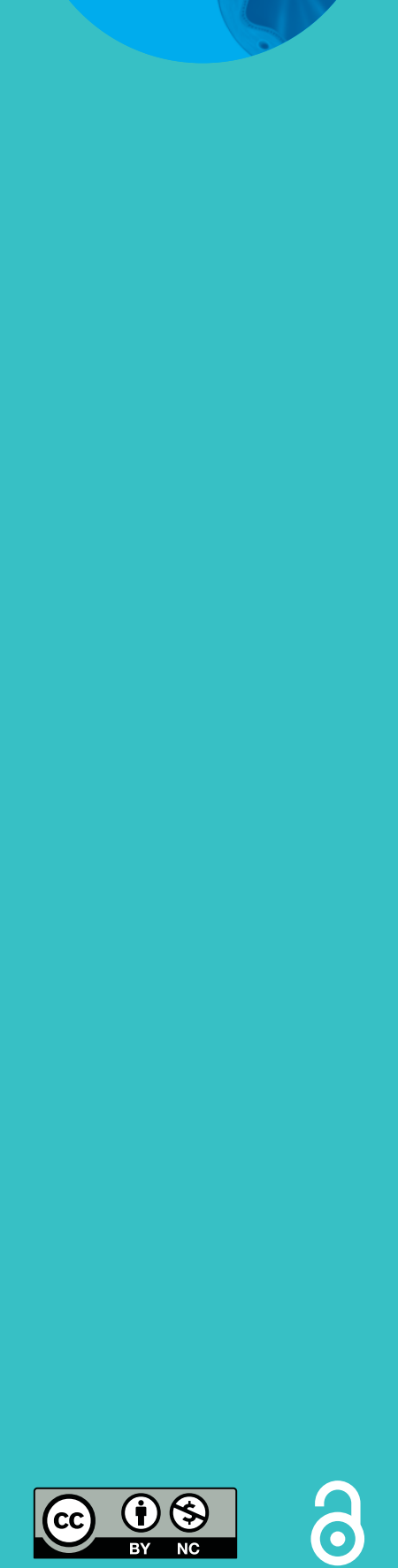

\section{Prevalence of comorbidities and impact on pulmonary rehabilitation outcomes}

\author{
Stacey J. Butler (1) ${ }^{1}$, Lok Sze Katrina Li (1) ${ }^{1,2}$, Lauren Ellerton', \\ Andrea S. Gershon (10 ${ }^{1,3,4,5,6}$, Roger S. Goldstein (1) ${ }^{1,5}$ and Dina Brooks ${ }^{1,5,7,8}$
}

Affiliations: 'Dept of Respiratory Medicine, West Park Healthcare Centre, Toronto, ON, Canada. ${ }^{2}$ School of Health Sciences, University of South Australia, Adelaide, Australia. ${ }^{3}$ Sunnybrook Research Institute, Toronto, ON, Canada. ${ }^{4}$ Institute of Health Policy, Management and Evaluation, University of Toronto, Toronto, ON, Canada. ${ }^{5}$ Institute of Medical Sciences, University of Toronto, Toronto, ON, Canada. ${ }^{6}$ Dept of Medicine, University of Toronto, Toronto, ON, Canada. ${ }^{7}$ School of Rehabilitation Sciences, McMaster University, Hamilton, ON, Canada. ${ }^{8}$ Rehabilitation Sciences, University of Toronto, Toronto, ON, Canada.

Correspondence: Stacey J. Butler, Dept of Respiratory Medicine, West Park Healthcare Centre, 82 Buttonwood Avenue, Toronto, Ontario, Canada M6P 1A1. E-mail: stacey.butlerQutoronto.ca

\section{ABSTRACT}

Background: The presence of comorbid conditions could impact performance in pulmonary rehabilitation (PR) programmes. We aimed to compare the comorbidity prevalence among those with chronic obstructive pulmonary disease (COPD) and interstitial lung disease (ILD) and evaluate the impact on PR response.

Methods: We performed a retrospective cohort study, recording comorbidities for all patients with COPD or ILD referred to PR. Participants were classified as responders to PR if they met the minimal important difference for exercise capacity and health-related quality of life (HRQoL). The prevalence of comorbidities and impact on PR outcomes were compared by lung disease and by sex using a univariate analysis and multivariate logistic regression.

Results: The mean number of comorbidities was similar among those with COPD $(3.3 \pm 2.1, \mathrm{n}=242)$ and ILD $(3.2 \pm 1.9, n=66) \quad(p>0.05)$. Females had a higher number of comorbidities than males in both COPD $(\mathrm{p}=0.001)$ and ILD $(\mathrm{p}=0.017)$ populations. Circulatory $(64 \%)$ and endocrine/metabolic $(45 \%)$ conditions were most common in COPD. In ILD, digestive (55\%) and circulatory (53\%) comorbidities were most prevalent. In people with ILD, those over 65 years, with musculoskeletal/connective tissue disease or circulatory disease were less likely to obtain meaningful improvements in exercise capacity. There was no impact of comorbidities on exercise capacity in COPD or on HRQoL in ILD.

Conclusions: The majority of patients with COPD or ILD enrolled in PR programmes have multiple comorbidities that may affect improvements in exercise capacity. PR programmes may be less effective for older adults with ILD and comorbid circulatory or musculoskeletal disease.

@ERSpublications

Comorbidities are common in people with chronic lung disease and can affect their ability to exercise. People with COPD have a similar comorbidity profile to people with interstitial lung disease (ILD). People with ILD and musculoskeletal disease or circulatory conditions are less likely to improve their exercise capacity after pulmonary rehabilitation. http://bit.ly/2p 8 WSIn

Cite this article as: Butler SJ, Li LSK, Ellerton L, et al. Prevalence of comorbidities and impact on pulmonary rehabilitation outcomes. ERJ Open Res 2019; 5: 00264-2019 [https://doi.org/10.1183/ 23120541.00264-2019].

This article has supplementary material available from openres.ersjournals.com.

Received: 16 May 2019 | Accepted after revision: 7 Oct 2019

Copyright $\odot$ ERS 2019. This article is open access and distributed under the terms of the Creative Commons Attribution Non-Commercial Licence 4.0. 


\section{Introduction}

Chronic respiratory diseases are an important cause of morbidity and mortality worldwide [1]. They are characterised by symptoms of breathlessness, fatigue and poor health-related quality of life (HRQoL) [2-4]. Pulmonary rehabilitation (PR) programmes are the standard of care for individuals with chronic respiratory disease such as chronic obstructive pulmonary disease (COPD) [5] and interstitial lung disease (ILD) [6].

PR combines supervised and personalised exercise training with education and self-management strategies [6]. Strong evidence supports improvements following PR on exercise capacity, HRQoL and symptoms such as dyspnoea and fatigue for people with COPD [7] and ILD [8]. Upon admission to PR programmes, many patients with COPD present with multiple comorbidities; however, their prevalence estimates vary depending on how comorbidities are reported. Self-reporting suggests a prevalence of at least one comorbidity in $50-60 \%$ of patients $[9,10]$, whereas objectively identifying comorbidities has shown $97 \%$ of patients with COPD entering PR had at least one comorbidity, with 53.5\% having four or more [11]. Common comorbidities in the COPD population include but are not limited to; cardiovascular disease, musculoskeletal disease (osteoarthritis and osteoporosis), lung cancer and metabolic disease (diabetes) [12-14]. In the ILD population, cardiac and gastrointestinal comorbidities (gastro-oesophageal reflux disease (GORD)), along with pulmonary comorbidities are common [15]. Shared risk factors for the development of respiratory disease and common comorbidities include age, cigarette smoking, physical inactivity and an unhealthy diet [16]. Sex differences for comorbidity prevalence have been noted in general COPD cohorts [17], but limited information is available on the presence of comorbidities by sex in those with COPD or ILD enrolled in PR.

Most information available is in regard to those with COPD. A systematic review by Hornikx et al. [18] noted less improvement following PR in individuals with COPD and comorbid behavioural, cardiovascular or musculoskeletal disorders, although the review only included four studies. To the best of our knowledge, there is no information on how comorbidities influence PR in those with ILD. We conducted this retrospective study to determine the prevalence of comorbidities and the impact on PR outcomes for individuals with ILD compared with those with COPD.

\section{Methods}

\section{Study design and participants}

We performed a retrospective cohort study of all patients with COPD or ILD enrolled in the inpatient and outpatient PR programmes at West Park Healthcare Centre from January 1, 2016 to December 31, 2017. Patients were selected according to the following inclusion criteria: 1) enrolled in inpatient or outpatient $P R, 2)$ physician diagnosis of COPD or ILD according to medical records, 3) over the age of 40.

Diagnosis was confirmed from the most recent spirometry testing. In the COPD population (forced expiratory volume in $1 \mathrm{~s}\left(\mathrm{FEV}_{1}\right) /$ forced vital capacity $\left.(\mathrm{FVC})<70 \%\right)$, disease severity was also determined by spirometry. Demographic and other information was extracted from the patients' admission physician and nursing notes.

The study received ethics approval from the Joint Bridgepoint Health (West Park Healthcare Centre) Toronto Central Community Care Access Centre, Toronto Grace Health Centre Research Ethics Board.

\section{Pulmonary rehabilitation}

All participants attended a standard PR programme at West Park Healthcare Centre. Participants in the outpatient PR programme attend twice weekly for 10 weeks with each session consisting of breathing exercises $(30 \mathrm{~min})$, education classes $(1 \mathrm{~h})$ and supervised exercise $(\sim 45 \mathrm{~min}-1 \mathrm{~h}$ of strength and aerobic training on alternating days). After 2 weeks all patients are provided with a home exercise programme and advised to exercise at least 4 days a week in total. Participants in the inpatient PR programme attend 5 days a week for 4-6 weeks and receive the same breathing exercises and number of education classes as the outpatient programme. Inpatients exercise twice a day, 5 days a week for a more intensive programme ( $\sim 45$ min -1 h of strength and aerobic training per session). Both programmes use standardised outcome measures at baseline and following completion of PR including the 6-minute walk test (6MWT) to measure functional exercise capacity, administered according to American Thoracic Society (ATS) guidelines [19], and the chronic respiratory disease questionnaire (CRQ) to measure HRQoL. We recorded these measures and whether the participant completed the PR programme, was discharged early and if so, the reason for early discharge.

\section{Comorbidities}

Comorbidities were extracted from the patients' medical records through their detailed PR admission assessment. Individual comorbidities were recorded and converted to comorbidity categories according to 
the International Statistical Classification of Diseases and Related Health Problems 10th Revision (ICD-10). A list of all individual comorbidities considered is provided in supplementary table 1. Participants were grouped according to the number of comorbidities (0-2, 3-4 and 5 or more). Prevalence of each comorbid condition and the corresponding comorbidity category was determined overall and by sex for the COPD and ILD populations.

\section{Outcomes}

The primary outcome was to determine the impact of comorbidities on PR response in terms of exercise capacity (6-minute walk distance (6MWD)) and HRQoL (CRQ domains). The secondary outcome was to determine the prevalence of comorbidities by the type of lung disease and sex. The PR outcomes (6MWD and CRQ domains) were treated as binary dependent variables in statistical models (responders and nonresponders). To distinguish responders from nonresponders, the minimal important differences (MID) were as follows; $30 \mathrm{~m}$ for 6MWD [20] and 0.5 units in CRQ domains [21]. First, a univariate analysis (Chi-squared test) was used to analyse correlations between PR outcomes and demographics, comorbidity category or number of comorbidities. Any significant findings were included in a multivariate logistic regression model to determine the predictive role on PR outcomes. Co-linearity was defined as variance inflation factor $>5$, condition index $>15$, tolerance $<0.2$ ). All results were considered to be statistically significant if $\mathrm{p}<0.05$.

\section{Statistics}

Statistical analyses were performed using IBM SPSS Statistics for Windows (version 25.0, Armonk, NY, USA). Results are expressed as percentages and means (SD). Comparison of comorbidity prevalence between males and females was made using the Chi-squared test.

\section{Results}

A total of 308 patients with COPD $(n=242)$ and ILD $(n=66)$ were admitted to the PR programme and met the inclusion criteria during the study period. There were 230 inpatients (75\%) and 78 outpatients $(25 \%)$. Demographic and baseline characteristics for the COPD and ILD cohorts are provided in table 1. Smoking history was available for $87 \%$ of the study population. The COPD cohort had a significantly higher smoking history compared with the ILD cohort $(\mathrm{p}<0.001)$ and there were more lifelong never-smokers in the ILD group $(p<0.001)$. Those with ILD were younger than the COPD cohort $(p=0.009)$ and there was a higher percentage of oxygen users $(p=0.001)$. Within the COPD cohort, females were more likely to use supplemental oxygen $(\mathrm{p}=0.01)$ and gait aids $(\mathrm{p}=0.007)$ compared with males. Females with COPD also had higher modified Medical Research Council Dyspnoea Scale (mMRC) scores $(p=0.028)$, lower smoking history $(\mathrm{p}=0.02)$ and lower total scores on the CRQ at admission $(\mathrm{p}=0.005)$

In the COPD population, the majority had severe (Global Initiative for Chronic Obstructive Lung Disease (GOLD) 3: 45\%) or very severe airflow limitation (GOLD 4: 35\%). The ILD population consisted of individuals with idiopathic pulmonary fibrosis (IPF), sarcoidosis, nonspecific interstitial pneumonia and unspecified ILD. Spirometry results were available for $75.8 \%$ of the ILD population (FVC \%

\begin{tabular}{|c|c|c|c|c|c|c|}
\hline Characteristics & \multicolumn{3}{|c|}{ COPD } & \multicolumn{3}{|c|}{ ILD } \\
\hline Age years & $70.2 \pm 9.2$ & $71.2 \pm 9.0$ & $69.3 \pm 9.4$ & $66.7 \pm 10.7^{* *}$ & $66.6 \pm 9.9$ & $66.9 \pm 11.5$ \\
\hline mMRC score & $3.2 \pm 0.8(n=190)$ & $3.3 \pm 0.8^{\#}(n=93)$ & $3.0 \pm 0.9(n=97)$ & $3.3 \pm 0.9(n=49)$ & $3.1 \pm 0.8(n=22)$ & $3.4 \pm 1.0(n=27)$ \\
\hline Smoking history pack-years & $47.0 \pm 23.9$ & $42.2 \pm 21.2^{\# \#}$ & $52.0 \pm 25.5$ & $33.6 \pm 18.7^{* * *}$ & $32.3 \pm 8.0^{\#}$ & $34.2 \pm 22.2$ \\
\hline Never-smokers & $3.3 \%$ & $5.0 \%$ & $1.6 \%$ & $17 \% * * *$ & $29 \%$ & $5.7 \%$ \\
\hline 6MWD \% predicted & $44.3 \pm 17.0$ & $44.7 \pm 16.8$ & $43.9 \pm 17.3$ & $40.1 \pm 16.7$ & $36.7 \pm 16.0$ & $43.3 \pm 17.0$ \\
\hline 6MWD m & $271.4 \pm 105.9$ & $259.4 \pm 99.6$ & $282.8 \pm 110.7$ & $255.0 \pm 111.3$ & $222.9 \pm 97.2^{\#}$ & $286.0 \pm 116.7$ \\
\hline CRQ total score & $4.0 \pm 1.1$ & $3.7 \pm 1.1^{\# \#}$ & $4.2 \pm 1.0$ & $4.0 \pm 1.1$ & $3.9 \pm 1.1$ & $4.2 \pm 1.1$ \\
\hline
\end{tabular}


FIGURE 1 Number of comorbid conditions in the study cohort of patients admitted to pulmonary rehabilitation.

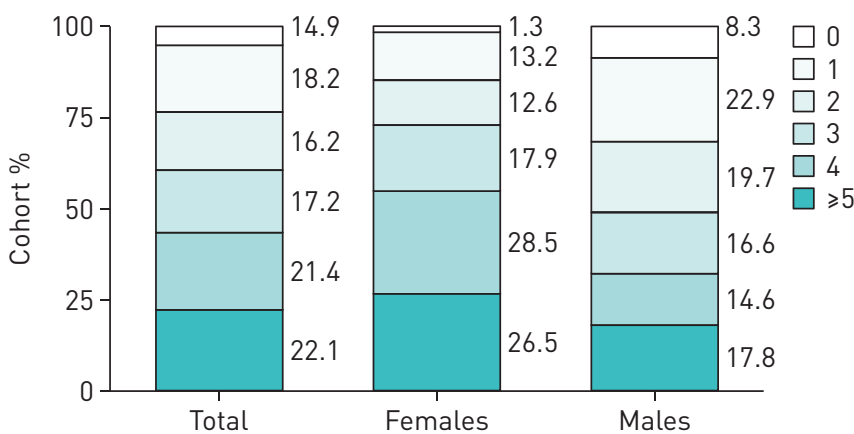

predicted $=57.7 \pm 18.8, \mathrm{FEV}_{1} / \mathrm{FVC}=76.0 \pm 18.8$ ). Of the entire study population, $95 \%$ had at least one comorbid condition, with $61 \%$ having three or more (figure 1). The mean number of comorbidities was similar among those with COPD $(3.3 \pm 2.1)$ and ILD (3.2 \pm 1.9$)(p=0.973)$. Females had a higher number of comorbidities compared with males respectively, in both COPD (3.7 \pm 2.1 versus $2.8 \pm 2.0, \mathrm{p}=0.001)$ and ILD cohorts $(3.8 \pm 1.6$ versus $2.7 \pm 2.1, \mathrm{p}=0.017)$. The number of comorbidities ranged from $0-11$ for those with COPD and 0-9 for those with ILD.

The prevalence of comorbidity categories was similar among those with COPD (figure 2a) and those with ILD (figure 2b). A full list of individual comorbidities is provided in supplemental table 1. Circulatory conditions were the most common comorbidity in COPD (64\%). In the ILD population, digestive conditions were the most common (54\%) followed closely by circulatory conditions (53\%). Endocrine and metabolic conditions, musculoskeletal and connective tissue diseases and mental health disorders were also common (figure 2), with similar prevalence among those with COPD or ILD ( $>0.05)$ (see supplemental table 2). Within the COPD cohort, females were more likely than males to have digestive comorbidities ( $52 \%$ versus $37 \%, \mathrm{p}=0.028)$, musculoskeletal and connective tissue conditions $(50.0 \%$ versus $34 \%, \mathrm{p}=0.013$ ) and mental and behavioural conditions ( $50 \%$ versus $24 \%, \mathrm{p}=0.000)$. Within the ILD cohort, females were more likely than males to have musculoskeletal and connective tissue conditions (55\% versus $26 \%$, $\mathrm{p}=0.023$ ) and mental and behavioural conditions ( $71 \%$ versus $20 \%, \mathrm{p}=0.000)$.

Programme completion rates were similar for inpatients $(74 \%)$ and outpatients $(77 \%, \mathrm{p}=0.762)$ and those with COPD (75\%) and ILD (76\%, p=1.00). Reasons for early discharge included acute exacerbations, nonrespiratory health events and personal reasons. Overall, $66 \%$ of patients in the entire study population met the MID of $30 \mathrm{~m}$ for the 6MWD (65\% of those with COPD and 69\% of those with ILD, p=0.735).
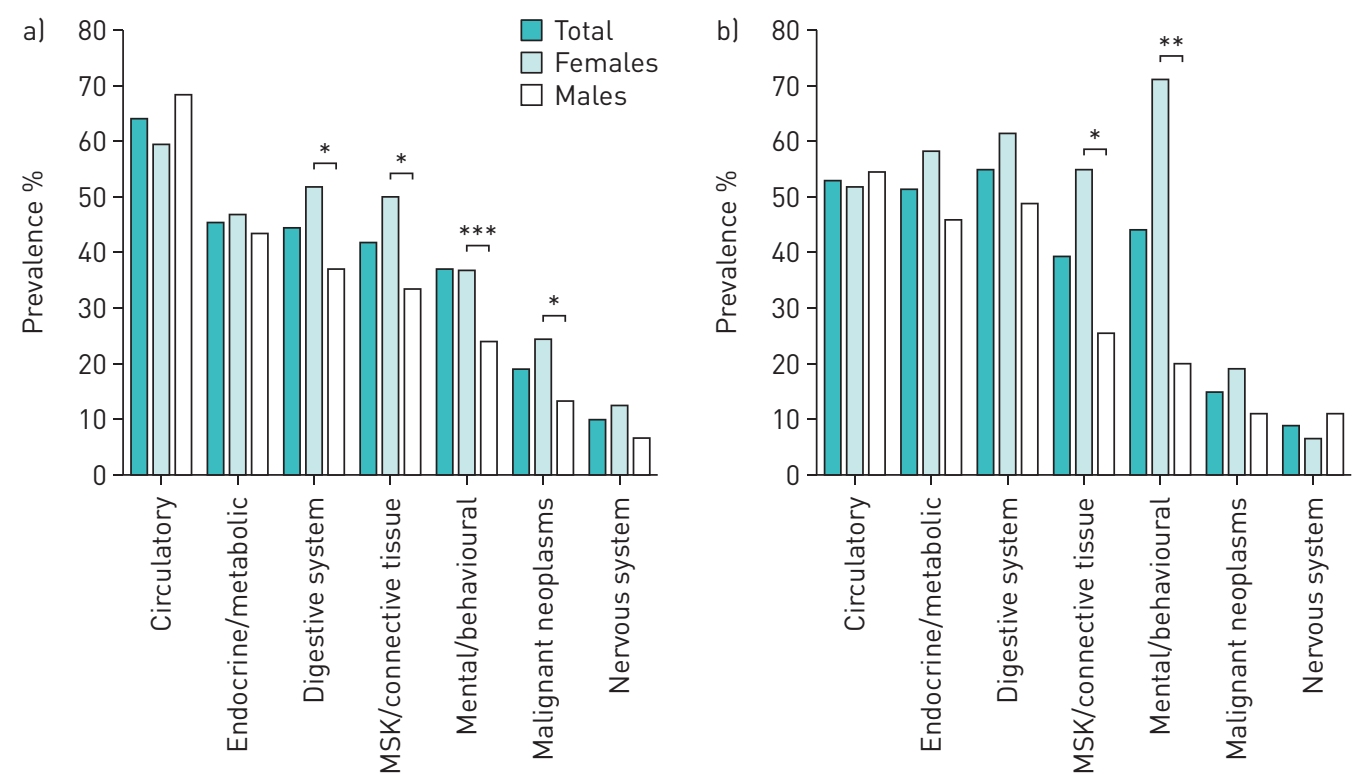

FIGURE 2 Prevalence of types of comorbidities in individuals with a) COPD and b) interstitial lung disease attending pulmonary rehabilitation. Chi-squared test of comorbidities between females and males. ${ }^{*}: p<0.05$; ${ }^{* *}: p<0.01 ;{ }^{* * *}: p<0.001$ 
The majority of the study population met the MID of 0.5 units for improvement in total CRQ score with no differences between the COPD and ILD cohorts (65\% versus $67 \%, \mathrm{p}=1.000$ ).

The results of a univariate analysis for the impact of the number and type of comorbidities on improvement in exercise capacity (6MWD), is depicted in table 2. In the ILD cohort, improvement in exercise capacity was impacted by age $(p=0.003)$ and diseases of the circulatory $(p=0.027)$ and musculoskeletal systems $(\mathrm{p}=0.013)$. In contrast, in the COPD subcohort, there were no significant associations between demographics, type or number of comorbidities and response to PR in terms of exercise capacity.

There was no significant impact of comorbidities on meeting the MID improvement in total CRQ score (table 3). Within the COPD cohort, sex impacted total CRQ score improvement, with more females improving compared with males $(p=0.036)$. Significantly associated variables from the univariate analysis were entered into a multivariate logistic regression model (table 4). People with ILD who did not have comorbid circulatory $(\mathrm{OR}=7.8,95 \% \mathrm{CI}: 1.1-54.9, \mathrm{p}=0.040)$ or musculoskeletal and connective tissue disease $(\mathrm{OR}=7.8,95 \% \mathrm{CI}: 1.3-47.1, \mathrm{p}=0.025)$ and were younger than 65 years $(\mathrm{OR}=11.0,95 \% \mathrm{CI}$ : $1.0-118.9, \mathrm{p}=0.048$ ) were more likely to have meaningful improvement in exercise capacity following PR. There was no impact of comorbidities on meaningful improvement in HRQoL and thus a multivariate regression analysis was not performed for this variable.

TABLE 2 Impact of demographics, and number and type of comorbidities on achieving minimal important difference in exercise capacity

\begin{tabular}{|c|c|c|}
\hline Demographic & COPD $(n=193)$ & ILD ( $n=48)$ \\
\hline \multicolumn{3}{|l|}{ Age group } \\
\hline$<65$ years & $74.5 \%$ & $94.7 \% * *$ \\
\hline$\geqslant 65$ years & $61.6 \%$ & $51.7 \%$ \\
\hline \multicolumn{3}{|l|}{ mMRC score } \\
\hline $0-1$ & $71.4 \%$ & $100 \%$ \\
\hline 2 & $46.4 \%$ & $33.3 \%$ \\
\hline $3-4$ & $68.1 \%$ & $70.0 \%$ \\
\hline \multicolumn{3}{|l|}{ Sex } \\
\hline Female & $67.4 \%$ & $69.6 \%$ \\
\hline Male & $63.5 \%$ & $68.0 \%$ \\
\hline \multicolumn{3}{|l|}{ Oxygen use } \\
\hline Yes & $66.3 \%$ & $76.9 \%$ \\
\hline No & $64.2 \%$ & $65.7 \%$ \\
\hline \multicolumn{3}{|c|}{ Type of comorbidity } \\
\hline \multicolumn{3}{|c|}{ Circulatory } \\
\hline Yes & $63.3 \%$ & $53.8 \% *$ \\
\hline No & $69.2 \%$ & $86.4 \%$ \\
\hline \multicolumn{3}{|l|}{ Digestive } \\
\hline Yes & $65.9 \%$ & $65.4 \%$ \\
\hline No & $64.8 \%$ & $72.7 \%$ \\
\hline \multicolumn{3}{|c|}{ Endocrine and metabolic } \\
\hline Yes & $66.3 \%$ & $62.5 \%$ \\
\hline No & $64.4 \%$ & $75.0 \%$ \\
\hline \multicolumn{3}{|c|}{ MSK and connective tissue } \\
\hline Yes & $59.5 \%$ & $47.4 \% *$ \\
\hline No & $69.3 \%$ & $82.8 \%$ \\
\hline \multicolumn{3}{|c|}{ Mental and behavioural } \\
\hline Yes & $67.1 \%$ & $65.2 \%$ \\
\hline No & $64.3 \%$ & $72.0 \%$ \\
\hline \multicolumn{3}{|c|}{ Number of comorbidities } \\
\hline $0-2$ & $65.8 \%$ & $87.5 \%$ \\
\hline $3-4$ & $70.7 \%$ & $58.3 \%$ \\
\hline$\geqslant 5$ & $54.8 \%$ & $62.5 \%$ \\
\hline \multicolumn{3}{|c|}{$\begin{array}{l}\text { Data are presented as the percentage of responders achieving an increase in } 6 \text {-min walking distance } \\
\geqslant 30 \text { m. ILD: interstitial lung disease; mMRC: modified Medical Research Council dyspnoea scale; MSK: } \\
\text { musculoskeletal. *: } p<0.05 ;{ }^{* *}: p<0.01 \text {. }\end{array}$} \\
\hline
\end{tabular}


TABLE 3 Impact of demographics, number and type of comorbidity on achieving minimal important difference in health-related quality of life

\begin{tabular}{|c|c|c|}
\hline Demographic & COPD $(n=144)$ & ILD $(n=36)$ \\
\hline \multicolumn{3}{|l|}{ Age group } \\
\hline$<65$ years & $73.8 \%$ & $70.6 \%$ \\
\hline$\geqslant 65$ years & $61.8 \%$ & $63.2 \%$ \\
\hline \multicolumn{3}{|l|}{ mMRC score } \\
\hline $0-1$ & $28.6 \%$ & $50.0 \%$ \\
\hline 2 & $63.6 \%$ & $100.0 \%$ \\
\hline $3-4$ & $71.7 \%$ & $64.0 \%$ \\
\hline \multicolumn{3}{|l|}{ Sex } \\
\hline Female & $74.3 \% *$ & $81.3 \%$ \\
\hline Male & $56.8 \%$ & $55.0 \%$ \\
\hline \multicolumn{3}{|l|}{ Oxygen use } \\
\hline Yes & $61.6 \%$ & $72.4 \%$ \\
\hline No & $69.0 \%$ & $43.0 \%$ \\
\hline \multicolumn{3}{|c|}{ Type of comorbidity } \\
\hline \multicolumn{3}{|c|}{ Circulatory } \\
\hline Yes & $60.4 \%$ & $73.7 \%$ \\
\hline No & $75.0 \%$ & $58.8 \%$ \\
\hline \multicolumn{3}{|l|}{ Digestive } \\
\hline Yes & $69.7 \%$ & $61.9 \%$ \\
\hline No & $61.5 \%$ & $73.3 \%$ \\
\hline \multicolumn{3}{|c|}{ Endocrine and metabolic } \\
\hline Yes & $62.0 \%$ & $82.4 \%$ \\
\hline No & $68.5 \%$ & $52.6 \%$ \\
\hline \multicolumn{3}{|c|}{ MSK and connective tissue } \\
\hline Yes & $67.8 \%$ & $69.2 \%$ \\
\hline No & $63.5 \%$ & $65.2 \%$ \\
\hline \multicolumn{3}{|c|}{ Mental and behavioural } \\
\hline Yes & $70.2 \%$ & $70.6 \%$ \\
\hline No & $62.9 \%$ & $63.2 \%$ \\
\hline \multicolumn{3}{|c|}{ Number of comorbidities } \\
\hline $0-2$ & $69.0 \%$ & $64.3 \%$ \\
\hline $3-4$ & $64.3 \%$ & $58.8 \%$ \\
\hline$\geqslant 5$ & $60.0 \%$ & $100 \%$ \\
\hline
\end{tabular}

\section{Discussion}

We conducted a retrospective cohort study and found that people enrolled in a PR programme with ILD had a similar number of comorbidities as people with COPD. The majority of patients presented with diseases of the circulatory system, and over $40 \%$ had gastrointestinal, endocrine and metabolic, musculoskeletal and connective tissue or mental and behavioural conditions. Despite having a number of comorbid conditions, most patients with COPD and ILD still improved their exercise capacity and HRQoL following PR. However, in people with ILD, the presence of musculoskeletal and connective tissue disease or circulatory conditions was associated with a lower impact of PR on improvements in exercise capacity. Comorbid conditions should be taken into account during admission assessments for PR in order to provide patients with a personalised exercise programme that will result in optimal improvements in exercise capacity.

A previous systematic review of individuals with COPD in PR concluded that osteoporosis was associated with reduced improvement in exercise capacity, that cardiovascular disease was negatively associated with improvement in quality of life and that anxiety and depression impacted the ability to improve dyspnoea scores [18]. We did not find any associations between comorbidities and PR outcomes in our COPD cohort, despite having a higher prevalence of hypertension, dyslipidaemia, osteoarthritis and osteoporosis compared with previous studies [9,10]. This is in keeping with the findings of Mesquita et al. [22] where the number and type of comorbidities had no impact on PR outcomes in a COPD cohort. It is important to note that previous studies in this area included hypertension as a metabolic disease and thus the 


\begin{tabular}{|c|c|c|c|c|c|}
\hline & Dependent variable & Independent variable & $\beta \pm \mathrm{SE}$ & $e^{\beta}(95 \% C l)$ & p-value \\
\hline \multirow[t]{3}{*}{ Unadjusted analysis } & 6MWD improvement (MID) & No circulatory comorbidity & $2.051 \pm 0.997$ & $7.775(1.102-54.863)$ & 0.040 \\
\hline & & No MSK/connective tissue comorbidity & $2.055 \pm 0.917$ & $7.807(1.293-47.124)$ & 0.025 \\
\hline & & Age $<65$ years & $2.402 \pm 1.212$ & $11.041(1.026-118.854)$ & 0.048 \\
\hline Adjusted $^{\#}$ analysis & & Age $<65$ years & $2.415 \pm 1.338$ & $11.189(0.813-153.960)$ & 0.071 \\
\hline
\end{tabular}

6MWD: 6-min walk distance; MID: minimal important difference; MSK: musculoskeletal. " : for sex, oxygen use and modified Medical Research Council dyspnoea scale score. Bold indicates statistically significant p-values.

prevalence rates for metabolic conditions differed in our study, where hypertension was considered a circulatory disease according to the ICD-10 coding system.

Previous studies have reported that comorbidities are common in people with COPD attending PR programmes [12]. However, there is much less information regarding comorbidities in patients with ILD. Pulmonary comorbidities, such as emphysema, lung cancer and pulmonary hypertension are commonly recognised in people with IPF [23] but less attention has been given to the prevalence of nonpulmonary conditions. A common nonpulmonary comorbidity in IPF is GORD [23] and a recent Medicare study suggested that diseases of the circulatory system, including hypertension and congestive heart failure, and endocrine or metabolic diseases including type II diabetes, are also common in the IPF population [24]. The systemic nature of ILD in many people suggests other comorbidities may affect the ability of patients with ILD to improve in PR programmes. To our knowledge, this is the first study to report the prevalence of comorbidities in people with ILD attending PR and explore the impact of comorbidities on PR outcomes. The prevalence of comorbidities in the ILD population was similar to the COPD population, with a mean of three comorbid conditions per patient. Musculoskeletal diseases such as osteoporosis and osteoarthritis were significantly more prevalent in females with ILD compared with males. We found both musculoskeletal and connective tissue disease and diseases of the circulatory system negatively impacted the ability to improve exercise capacity in the ILD population. Additionally, those of younger age were more likely to improve exercise capacity. However, the role of dyspnoea should not be ignored as evident in the adjusted analysis. As with COPD, a personalised approach to PR may maximise improvements in patients with ILD, particularly among those with comorbid circulatory or musculoskeletal disease.

We report differences in comorbidities by sex in people with COPD or ILD enrolled in PR programmes. A number of comorbidities were more prevalent in females compared with males. Conditions, such as hypothyroidism and osteoporosis, particularly post-menopause, are known to be more prevalent in females in the general population $[25,26]$. Anxiety and depression were significantly higher for females in both people with COPD and ILD. Previous research has also noted a higher prevalence of anxiety and depression in women compared with men with COPD, despite similar disease characteristics and dyspnoea ratings [27]. In ILD populations, anxiety and depression are common, but there does not appear to be a sex difference in prevalence when measured prospectively $[28,29]$. In this retrospective study we found a significantly higher prevalence of mental health disorders in females with ILD.

Healthcare professionals in $\mathrm{PR}$ may need to individualise the PR programme in the presence of comorbidities, particularly musculoskeletal conditions. Alternative forms of PR, such as water-based training for individuals with musculoskeletal conditions [30] have shown significant improvements in exercise capacity, dyspnoea and fatigue compared with traditional land-based training. Clinicians should be aware of the presence of coexisting cardiovascular disease among individuals with ILD who are enrolled in PR. There are a number of shared aspects between cardiac rehabilitation and PR, suggesting that a comprehensive cardiopulmonary rehabilitation programme could achieve benefits even when the two conditions coexist [31]. A simple and feasible step would be to offer an education component on cardiovascular disease for people with relevant coexisting conditions. An education component could also be developed for common endocrine and metabolic conditions, such as diabetes and would allow patients to have a better understanding of their condition and how to exercise safely.

The retrospective nature of this study is a limitation. We relied on conditions reported in medical records, which may be an underestimate of comorbidities. Additionally, patients may actually have been excluded from PR due to significant neurological, musculoskeletal or cardiac conditions, thus resulting in a lower 
prevalence of these conditions in the PR population compared with the general COPD or ILD population. An additional limitation is that this study was conducted at a single site where the healthcare team is accustomed to patients with severe disease and thus our results may not be generalisable to all centres with PR programmes. The ILD population in this study was small $(n=66)$, with only 48 patients having a post-PR 6MWT, which limits the statistical power in this group. Despite the small sample size, we still had statistically significant findings in the ILD population which suggests further exploration into the impact of nonpulmonary comorbidities in this disease population would be beneficial. The conclusions of this study should inform a prospective study of the influence of comorbidities in patients with chronic respiratory disease undergoing PR.

\section{Conclusion}

People with chronic respiratory disease who enroll in PR have a number of comorbid conditions, many of which are more prevalent in females. People with ILD have a similar number of comorbidities as those with COPD. Regardless of the type or number of comorbidities, PR can still improve HRQoL although older age and the presence of musculoskeletal conditions significantly impact the ability to obtain clinically important improvements in exercise capacity. Particularly of importance are older individuals with ILD who have comorbid circulatory or musculoskeletal disease. Individualised PR programmes that take comorbidities into account may offer further improvement in exercise capacity. Based on our findings, people with chronic respiratory disease should be offered PR if their comorbid conditions are stable and they can exercise safely.

Support statement: L.S.K. Li was supported by the Australia Awards Endeavour Research Fellowship funded by the Australian Government Dept of Education and Training. A.S. Gershon holds a Canadian Institute of Health Research (CIHR) New Investigator Award. D. Brooks holds a National Sanatorium Chair, West Park Healthcare Centre. The funding sources were not involved in the design of the study, or the collection, analysis or interpretation of data.

Conflict of interest: None declared.

\section{References}

1 World Health Organization. Global surveillance, prevention and control of chronic respiratory diseases : a comprehensive approach. Geneva, Switzerland, 2007.

2 Bateman ED, Hurd SS, Barnes PJ, et al. Global strategy for asthma management and prevention: GINA executive summary. Eur Respir J 2008; 31: 143-178.

3 Raghu G, Richeldi L. Current approaches to the management of idiopathic pulmonary fibrosis. Respir Med 2017; 129: 24-30.

4 Vogelmeier CF, Criner GJ, Martinez FJ, et al. Global Strategy for the Diagnosis, Management, and Prevention of Chronic Obstructive Lung Disease 2017 report: GOLD Executive Summary. Arch Bronconeumol 2017; 53: $128-149$.

5 O'Donnell DE, Aaron S, Bourbeau J, et al. Canadian Thoracic Society recommendations for management of chronic obstructive pulmonary disease - 2007 update. Can Respir J 2007; 14: Suppl. B, 5b-32b.

6 Spruit MA, Singh SJ, Garvey C, et al. An official American Thoracic Society/European Respiratory Society statement: key concepts and advances in pulmonary rehabilitation. Am J Respir Crit Care Med 2013; 188: e13-e64.

7 McCarthy B, Casey D, Devane D, et al. Pulmonary rehabilitation for chronic obstructive pulmonary disease. Cochrane Database Syst Rev 2015; 2: CD003793.

8 Dowman L, Hill CJ, Holland AE. Pulmonary rehabilitation for interstitial lung disease. Cochrane Database Syst Rev 2014; 10: CD006322.

9 Crisafulli E, Costi S, Luppi F, et al. Role of comorbidities in a cohort of patients with COPD undergoing pulmonary rehabilitation. Thorax 2008; 63: 487-492.

10 Crisafulli E, Gorgone P, Vagaggini B, et al. Efficacy of standard rehabilitation in COPD outpatients with comorbidities. Eur Respir J 2010; 36: 1042-1048.

11 Vanfleteren LEGW, Spruit MA, Groenen M, et al. Clusters of comorbidities based on validated objective measurements and systemic inflammation in patients with chronic obstructive pulmonary disease. Am J Respir Crit Care Med 2013; 187: 728-735.

12 Franssen FME, Rochester CL. Comorbidities in patients with COPD and pulmonary rehabilitation: do they matter? Eur Respir Rev 2014; 23: 131-141.

13 Spruit MA. Pulmonary rehabilitation. Eur Respir Rev 2014; 23: 55-63.

14 Corlateanu A, Covantev S, Mathioudakis AG, et al. Prevalence and burden of comorbidities in chronic obstructive pulmonary disease. Respir Investig 2016; 54: 387-396.

15 Margaritopoulos GA, Antoniou KM, Wells AU. Comorbidities in interstitial lung diseases. Eur Respir Rev 2017; 26: 160027.

16 Decramer M, Janssens W. Chronic obstructive pulmonary disease and comorbidities. Lancet Respir Med 2013; 1: 73-83.

17 Cazzola M, Bettoncelli G, Sessa E, et al. Prevalence of comorbidities in patients with chronic obstructive pulmonary disease. Respiration 2010; 80: 112-119.

18 Hornikx M, Van Remoortel H, Demeyer H, et al. The influence of comorbidities on outcomes of pulmonary rehabilitation programs in patients with COPD: a systematic review. Biomed Res Int 2013; 2013: 146148.

19 Brooks D, Solway S, Gibbons WJ. ATS statement on six-minute walk test. Am J Respir Crit Care Med 2003; 167 1287. 
Holland AE, Spruit MA, Troosters T, et al. An official European Respiratory Society/American Thoracic Society technical standard: field walking tests in chronic respiratory disease. Eur Respir J 2014; 44: 1428-1446.

21 Schunemann HJ, Puhan M, Goldstein R, et al. Measurement properties and interpretability of the chronic respiratory disease questionnaire (CRQ). COPD 2005; 2: 81-89.

22 Mesquita R, Vanfleteren LE, Franssen FM, et al. Objectively identified comorbidities in COPD: impact on pulmonary rehabilitation outcomes. Eur Respir J 2015; 46: 545-548.

23 Cano-Jimenez E, Hernandez Gonzalez F, Peloche GB. Comorbidities and complications in idiopathic pulmonary fibrosis. Med Sci (Basel) 2018; 6: E71.

24 Mortimer K, Hartmann N, Chan C, et al. Characterizing idiopathic pulmonary fibrosis patients using US Medicare-advantage health plan claims data. BMC Pulm Med 2019; 19 : 11.

25 Aoki Y, Belin RM, Clickner R, et al. Serum TSH and total T4 in the United States population and their association with participant characteristics: National Health and Nutrition Examination Survey (NHANES 1999-2002). Thyroid 2007; 17: 1211-1223.

26 Jackson RD, Mysiw WJ. Insights into the epidemiology of postmenopausal osteoporosis: the Women's Health Initiative. Semin Reprod Med 2014; 32: 454-462.

27 Laurin C, Lavoie KL, Bacon SL, et al. Sex differences in the prevalence of psychiatric disorders and psychological distress in patients with COPD. Chest 2007; 132: 148-155.

28 Holland AE, Fiore JF Jr, Bell EC, et al. Dyspnoea and comorbidity contribute to anxiety and depression in interstitial lung disease. Respirology 2014; 19: 1215-1221.

29 Lee YJ, Choi SM, Lee YJ, et al. Clinical impact of depression and anxiety in patients with idiopathic pulmonary fibrosis. PLoS ONE 2017; 12: e0184300.

30 McNamara RJ, McKeough ZJ, McKenzie DK, et al. Water-based exercise in COPD with physical comorbidities: a randomised controlled trial. Eur Respir J 2013; 41: 1284-1291.

31 Triest FJ, Singh SJ, Vanfleteren LE. Cardiovascular risk, chronic obstructive pulmonary disease and pulmonary rehabilitation: Can we learn from cardiac rehabilitation? Chron Respir Dis 2016; 13: 286-294. 